\title{
Addition of Lab Experiences to a Course in Dynamic Systems with Vibrations
}

\section{Dr. Diane L. Peters PE, Kettering University}

Dr. Peters is an Assistant Professor of Mechanical Engineering at Kettering University.

\section{Dr. Janet Brelin-Fornari, Kettering University}

Dr. Brelin-Fornari is a professor of Mechanical Engineering and the Director of the Kettering University Crash Safety Center. She began work in 1982 as an engineer with General Motors and moved to academia 1999. Dr. Brelin-Fornari teaches in the areas of dynamics, systems, and controls. She also conducts research in pediatric mobility safety. And, she brings both the classroom and the research together for students at the undergraduate and K-12 levels.

Ms. Sheryl Janca, Kettering University 


\title{
Addition of Lab Experiences to a Course in Dynamic Systems with Vibrations
}

\begin{abstract}
At Kettering University, all mechanical engineering students take a two-course sequence in Dynamic Systems; the first course is Dynamic Systems with Vibrations, and the second is Dynamic Systems with Controls. In the past, the first course has been purely a lecture class, while the second has included both lecture and lab time. Both courses were modified, as a result of student experiences and feedback from industry partners, with the lab redesigned for Dynamic Systems with Controls and a lab component involving Matlab/Simulink modeling and

simulations added to Dynamic Systems with Vibrations. This paper will focus on the lab addition to the first course, Dynamic Systems with Vibrations.

This paper will first present the background of the course and reasons for the addition of the lab, including the desired student outcomes. Next, it will discuss the initial design of the labs and the evolution to the current set of lab experiences. This discussion will include the key topics covered, the learning objectives of the labs, and the practical challenges in implementation. Finally, data from student instructor/course surveys will be presented, and future goals for the lab component of the class will be discussed.
\end{abstract}

\section{Background}

At Kettering University, all mechanical engineering majors take two courses in dynamic systems. In Dynamic Systems 1: Dynamic Systems with Vibrations (DSI), students learn about the bond graph modeling method for deriving a system's governing differential equations, and study the vibrations of mechanical systems, particularly second-order systems. In Dynamic Systems 2: Dynamic Systems with Controls (DSII), students study the characteristics of dynamic systems, given their differential equations, and learn about classical control topics. Initially, the Dynamic Systems 1 class was purely lecture-based, with four hours per week of lecture time. Dynamic Systems 2 was intended to be primarily a lab-based class, with four hours of laband two hours of lecture per week. However, much of the lab time for Dynamic Systems 2 was used for additional lecture.

Course evaluations suggested that students were not satisfied with their learning in these two classes. Two areas in particular were reevaluated: student's ability to use Matlab/Simulink to model and simulate dynamic systems and their ability to implement a practical controller on hardware. In order to improve the courses, the lecture and lab hours were re-allocated among the two courses, with a lab added to Dynamic Systems 1. After the change, both courses featured three lecture hours and two lab hours per week, and the existing lab for Dynamic Systems 2 was revamped completely, with a combination of Matlab/Simulink-based simulation labs and several hardware-based labs. ${ }^{1}$ The new Dynamic Systems 1 lab was designed to focus exclusively on Matlab/Simulink, with initial labs using Matlab and later labs incorporating Simulink.

Many approaches may be taken to learning Matlab, as it is a large software package that can be used for either simple applications or for very sophisticated programming, and in fact, can be the 
subject of a course all by itself. ${ }^{2}$ In DSI, the primary focus was on the dynamic systems course content, and the use of Matlab and Simulink were aimed at reinforcing core concepts in the course. Courses in dynamic systems have, at many institutions, been challenging for students; even those who were successful in their dynamics course may find the transition to dynamic systems to be difficult. ${ }^{3}$ The reasons for this difficulty may vary, and are not extensively studied, but they may be in part due to the need to integrate students' knowledge of differential equations with the engineering material that is presented. This is extremely important, however, and is in fact part of the Accreditation Board for Engineering and Technology (ABET) objectives for engineering programs (A. an ability to apply knowledge of mathematics, science and engineering, as well as K. an ability to use the techniques, skills, and modern engineering tools necessary for engineering practice). ${ }^{4}$

The development of the lab component of the course is subject to evaluation and improvement; evaluation of the lab is carried out through evaluation of students' performance, as well as through instructor-course evaluations. For this evaluation, Kettering University utilizes the Individual Development and Educational Assessment (IDEA) survey. This survey is widely used by many universities, ${ }^{5}$ and is often used to determine how well the ABET objectives A-K are being met. ${ }^{6}$ Many students choose not to fill out the evaluation, and therefore in small lab classes it is difficult to get statistically significant results; however, student comments are extremely useful, and are included in the evaluation of the labs.

\section{Initial Lab Design}

The first offering of the Dynamic Systems 1 lab course was in the Summer 2014 term. There were eight (8) labs in an 11-week term, as listed in Table 1.There was no lab during the week of mid-terms and the students were given two lab sessions to complete the final project.

\begin{tabular}{|c|l|}
\hline $\begin{array}{c}\text { Lab } \\
\text { Number }\end{array}$ & Lab Description \\
\hline 1 & $\begin{array}{l}\text { Introduction to Matlab and Solving } \\
\text { Differential Equations }\end{array}$ \\
\hline 2 & Matrix Math \\
\hline 3 & Spring Mass Damper System \\
\hline 4 & Linear Simulation in Matlab \\
\hline 5 & Introduction to Simulink \\
\hline 6 & Block Diagram Algebra \\
\hline 7 & First Order System Response \\
\hline & Final Lab Project: Modeling and \\
& Simulation of a More Complex System \\
\hline
\end{tabular}




\section{Lab 1: Solving Differential Equations}

In the first lab, students solved differential equations both analytically and using Matlab's symbolic solver, then used Matlab to plot the solutions. This lab served to also refresh the students on understanding the classification of differential equations. The focus was on $1^{\text {st }}$ and $2^{\text {nd }}$ order, homogeneous, linear equations with constant coefficients.Time was spent introducing students to the Matlab software, including the functions of the different workspaces of the software as well as several basic commands.

\section{Lab 2: Matrix Math}

The second lab focused on matrices.A review was provided on basic rules of matrix math including addition/subtraction, multiplication, symmetry, Identity matrix, determinants, and eigenvalues.Students learn to properly enter matrices in Matlab and then to solve various problems using Matlab commands including the "det" function and the "eig" function.

\section{Lab 3: Spring Mass Damper System}

The third lab focused on $2^{\text {nd }}$ order differential equation using a basic spring mass damper system.The students were asked to recall the "dsolve" command they learned in week one and the "subs" command was introduced to simulate different variations within the basic system.First the system included only the spring without damping. While keeping the spring value constant, students are asked to vary the mass and assess what effect the mass variation has on the amplitude and frequency of the system. They were then asked to hold the mass constant and vary the spring value and consider the same questions on amplitude and frequency. The third task in the lab was to add a damper to the simulation, again with varying values includingover dampened, under dampened and critically dampened.The deliverable of the lab was a graphical assessment of the displacement of the mass.

\section{Lab 4: Linear Simulation using Matlab}

In the fourth lab, the "lsim" command was introduced to the students. They started with the simple mass spring system from the prior lab and then a more complicated quarter-car suspension system with multiple degrees of freedom and outputs. Students were asked to graphically express the response of the system with a given input.

\section{Lab 5: Introduction to Simulink}

In this lab, students constructed a Simulink model representing a simple second-order system and performed calculations to find the damping required for resonance; they confirmed this value by simulating the system, and verifying that its response displayed resonance.Discussion with this lab included the use of Laplace Transforms to determine the transfer function of the system.They students became familiar with the various library tools used in Simulink and how the workspace is used to "build" simulations.

\section{Lab 6: Block Diagram Algebra}

This lab continued to build on the prior lab in that the students performed block diagram algebra on a simple block diagram, to find the transfer function. They then simulated the system both as 
the full block diagram in Simulink, and as the transfer function, and verified that the results were identical.Basic Simulink commands were reviewed and the students were required to graphically demonstrate the results of the simulations.

\section{Lab 7: First Order System Response}

In this lab, students created the dynamic model for a simple RC circuit, then simulated its response to a step input for the voltage. Concepts covered included the basic first-order step response, steady-state response, Final Value Theorem and the time constant.Bond graphs were covered in the lecture portion of the class and students utilized that knowledge to model the circuit.Block diagram and graphical outputs of the system were the deliverables for the lab.

\section{Lab 8: Final Lab Project}

In this project, students created a dynamic model of a more complex system than they had previously encountered. They then created a Simulink model for the system, and evaluated and plotted a number of different system responses. The project also included a comparison of the analytical description of the system to the simulation results. The goal of the final project was to include multiple concepts taught through the previous weeks. The culminating project included topics from both prior labs as well as lecture content. Students were asked to construct the simplified bond graph with causality, identify all governing system equations, develop the state equation, use Laplace transforms to determine the transfer function of the system, evaluate steady state response using Final Value Theorem, determine time constant and provide graphical outputs of the system.

\section{Evaluation and Improvement}

Students were given the opportunity to evaluate the course at the end of the term.Evaluations are anonymous and both the lecture and lab portions of the class have separate evaluations. While many students chose not to complete the evaluations, there were quite a few who did choose to make comments about the lab, and these were useful in determining whether the lab was a useful addition to the course. Overall, student responses were positive, indicating that they felt that they had deeper learning because of the addition of the lab. Some feedback from the evaluations included:

"There were some slight disconnects, but overall the lab worked well with the lecture."

"Getting re familiarized with Matlab was an advantage."

"The only thing I would put as a negative was if we had a lab assignment that covered material that we had not already covered in lecture. This made it more difficult to learn on a quick basis....."

"Excellent lab."

"Learning things I'll be able to apply during work." 
Evaluation of the lab based on the instructors' perspective indicated that students still had gaps in their ability to apply their knowledge, particularly in understanding how differential equations could be used to describe dynamic systems. Also, the evaluation of the lab topics on the final exam indicate that topics covered in the lab section had the lowest scores of graded problems.

\section{Discussion and Conclusion}

Overall the addition of a lab to the curriculum was well received by the students. They gained experience with the Matlab and Simulink software and recognize the connection between concepts taught in lecture and the simulations conducted in the lab.Some students had difficulty if the lab was ahead of the lecture when new concepts were introduced.Consideration must be taken when the labs rely on new content to ensure students have the basic information prior to the lab.

Since the scores on the lab topic problems on the final exam were the lowest of all topics evaluated, two strategies were implemented. First, homework assignments were given to give the students additional practice with computational methods. Second, quizzes were implemented in the lab to evaluate the lab-specific topics as well as a computer practicum. This will ensure that each student has an understanding of the topics and additional exposure prior to the final exam.

One of the other areas of evaluation for the lab is in the integration of the two courses, Dynamic Systems with Vibrations and Dynamic Systems with Controls. The perception of one of the instructors for the second course is that students are better prepared for the labs in the second course, having had exposure to Matlab and Simulink in the first course, but that has not yet been measured in any systematic way.

Work is on-going with respect to the lab details and handouts. Time constraints and balancing of topics within the lab continue to be addressed. Overall, three (3) instructors have taught the lecture portion of the course and six (6) have taught the lab portion. Continued feedback between the instructors has strengthened this new lab offering.

\section{References}

1. Peters, D.L., Stanley, R., Hoff, C. \& Casci, J. (2015) Redesign of Lab Experiences for a Senior Level Course in Dynamic Systems with Controls. Proceedings of the ASEE Annual Conference, Seattle, WA

2. Reckinger, S. M. \& Reckinger, S. J. (2014) An Interactive Programming Course Model for Mechanical Engineering Students. Proceedings of the ASEE Annual Conference, Indianapolis, IN.

3. Bedillion, M. D., Raisanen, R. J., \& Nizar, M. H. M. (2014) Improving Transitions Between Sophomore Dynamics and Junior DynamicSystems Courses. Proceedings of the ASEE Annual Conference, Indianapolis, IN.

4. Accreditation Board for Engineering and Technology. (2015). Criteria for Accrediting Engineering Programs, 2014 -2015. Retrieved from http://www.abet.org/wp-content/uploads/2015/05/E001-15-16EAC-Criteria-03-10-15.pdf 
5. Individual Development and Educational Assessment (IDEA) Center. (2016). Retrieved fromhttp://ideaedu.org/abouton 1/31/2016.

6. Garry, B. G. (2011). Relationship Between ABETTAC Criterion 3 A-K Student Learning Outcomes Achievement. Proceedings of the ASEE Annual Conference, Vancouver, Canada. 